\title{
Theory and Analysis of Electrode Size Optimization for Capacitive Microfabricated Ultrasonic Transducers
}

\author{
Ayhan Bozkurt, Igal Ladabaum, Abdullah Atalar, Senior Member, IEEE, \\ and Butrus T. Khuri-Yakub, Fellow, IEEE
}

\begin{abstract}
Theoretical analysis and computer simulations of capacitive microfabricated ultrasonic transducers indicate that device performance can be optimized through judicious patterning of electrodes. The conceptual basis of the analysis is that electrostatic force should be applied only where it is most effective, such as at the center of a circular membrane. If breakdown mechanisms are ignored, an infinitesimally small electrode with an infinite bias voltage results in the optimal transducer. A more realistic design example compares the 3-dB bandwidths of a fully metalized transducer and a partially metalized transducer, each tuned with a lossless Butterworth network. It is found that the bandwidth of the optimally metalized device is twice that of the fully metalized device.
\end{abstract}

\section{INTRODUCTION}

A IR-COUPLED and immersion capacitive ultrasonic transducers have existed for decades [1]-[3]. Recent developments in microfabrication technology have spurred new versions of the devices [4]-[10], and some theory explaining their operation has been proposed [4], [7], [11][13]. The main motivational forces behind transducer development are applications in air-coupled nondestructive evaluation (NDE) and in 3-D immersion imaging using 2$\mathrm{D}$ transducer matrices.

Although electrode patterning has been used for selective mode excitation of resonators [14] and in the optimization of capacitive pressure transducers and microphones [15], no group, to our knowledge, has used electrode patterning to optimize the performance of capacitive microfabricated ultrasonic transducers (cMUTs). In this paper, we present optimization criteria, analyses, and simulations that demonstrate that electrode patterning can be used to enhance the performance of cMUTs significantly.

Manuscript received February 18, 1998; accepted June 15, 1999. This project is sponsored by the United States Office of Naval Research.

A. Bozkurt and A. Atalar are with Bilkent University, Ankara, Turkey.

I. Ladabaum is with Sensant Corp., San Jose, CA.

B. T. Khuri-Yakub is with E. L. Ginzton Laboratory, Stanford University, Stanford, CA 94305.

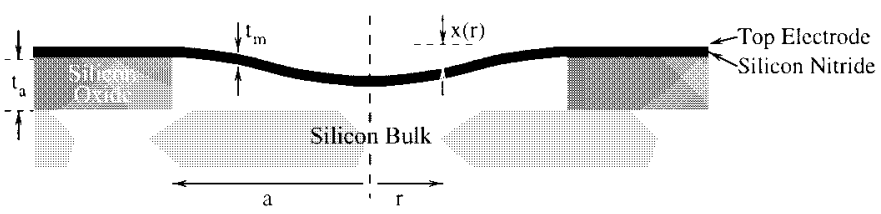

Fig. 1. Schematic view of an element of a silicon cMUT.

\section{Device And Circuit Model Description}

Techniques invented by the semiconductor industry are utilized to construct cMUTs [16]. Fig. 1 shows the crosssectional view of a typical cMUT element. The driving force for the deflection of the silicon nitride membrane is the electrostatic attraction between the metal top layer and the silicon substrate when a voltage is placed across them. Conversely, when acoustic pressure loads a biased membrane, its deflection is measured by either the flow of charge (constant voltage operation) or by voltage changes (constant charge operation) across the device.

The analysis of the cMUT structure is based on the equivalent circuit approach of Mason [17] as adapted in [4]. The model, asseen in Fig. 2, consists of a shunt input capacitance $C_{0}$ at the electrical port and an electromechanical transformer with turns ratio $1: n$. $Z_{a}$ is the lumped acoustic impedance of the membrane, and $Z_{l}$ is the acoustic load, which is just the acoustic impedance of the medium $Z_{\text {medium }}$ multiplied by the membrane area $S_{\text {membrane }}{ }^{1} V$ and $i$ show the input voltage and current, respectively. F is the total electrostatic force on the membrane under the assumption that electrostatic pressure is uniform at all points. For the lumped model, the measure for the membrane movement is its average velocity $v$.

When the transducer is loaded by a relatively high acoustic impedance medium (such as water), the acoustic impedance of the cMUT membrane $Z_{a}$ and the load $Z_{l}$ form a low quality factor circuit. In such cases, the membrane impedance can be neglected for frequencies near the mechanical resonance of the device. In [4], it is shown that the resonant impedance of typical MUT membranes is at least one order of magnitude below that of water. The

\footnotetext{
${ }^{1}$ Taking the acoustic load on the membrane to be simply $Z_{\text {medium }}$ multiplied by $S_{\text {membrane }}$ implies that the membrane vibration is planar. Although this is not strictly true for a single element, a collection of membranes does indeed behave as a piston transducer, as is evidenced in [4].
} 


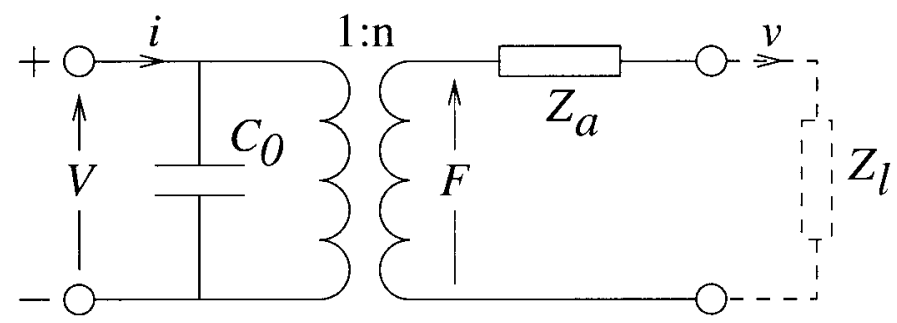

\section{Electrical Port Acoustic Port}

Fig. 2. Equivalent electrical circuit for the cMUT membrane.

equivalent circuit then reduces to a simpler network containing $Z_{l}, C_{0}$, and the electro-mechanical transformer. For clarity, we limit ourselves to analysis based on the reduced immersion cMUT model of Fig. 3. However, it should be noted that the principle of electrode size optimization is applicable to cMUTs when the mechanical impedance of the membrane is significant.

\section{Optimization Criteria AND PARAmeters}

The ideal ultrasonic transducer would have infinite bandwidth and no insertion loss. A practical optimization guideline for cMUTs is to maximize their bandwidth at an insertion loss smaller than $3 \mathrm{~dB}$. In this discussion, we neglect contributions to insertion loss that stem from parasitic real losses in the transducer, such as membrane heating, acoustic radiation into the supporting structure, and losses in the transducer cavity. We also assume that driving and receiving electronics can be designed to maximize power transfer for the transducers of interest. In short, we limit our discussion to the effect of the shunt capacitance $C_{0}$ on the efficiency and bandwidth of the device. If bandwidth were not a concern, one could simply tune out $C_{0}$ with an inductor to achieve zero insertion loss. But bandwidth is indeed a concern; so, a more complete formulation of the optimization objective is to minimize the time constant $\tau$ of the first-order network formed by the shunt input capacitance and the transformed radiation impedance, where $\tau=C_{0} Z_{l} / n^{2}$.

In minimizing $\tau$, we first consider the case of a fully metalized membrane with membrane area equal to electrode area $\left(S_{\text {membrane }}=S_{\text {electrode }}\right)$. We use expressions for $n_{\text {full }}$ and $C_{0}$ derived in [4] to obtain $\tau_{\text {full }}$ :

$$
\begin{gathered}
C_{0}=\frac{\epsilon_{0} \epsilon S_{\text {membrane }}}{\epsilon_{0} t_{m}+\epsilon t_{a}} \\
n_{\text {full }}=\frac{V_{D C} \epsilon_{0} \epsilon^{2} S_{\text {membrane }}}{\left(\epsilon_{0} t_{m}+\epsilon t_{a}\right)^{2}}
\end{gathered}
$$

where $V_{D C}$ is the bias across the device, $\epsilon$ is the permittivity of the membrane material, $\epsilon_{0}$ is the permittivity of air, $t_{m}$ is the membrane thickness, and $t_{a}$ is the air gap thickness. Thus,

$$
\tau_{\text {full }}=\frac{\left(\epsilon_{0} t_{m}+\epsilon t_{a}\right)^{3}}{V_{D C}^{2} \epsilon_{0} \epsilon^{3}} Z_{\text {medium }}
$$

It is clear that to minimize $\tau_{\text {full }}, V_{D C}$ should be made as large as possible, and $t_{m}$ and $t_{a}$ should be made as small as possible. Theoretical and practical limits to such minimization exist. The fabrication process has thickness tolerances on the order of hundreds of angstroms; so, vertical dimensions of less than a few thousand angstroms could not be fabricated repeatably with the existing technology. Many applications also preclude the use of very high voltages because it is too dangerous or too costly to provide the high voltage. More significant, however, are the fundamental limitations, which are set by breakdown fields of the materials comprising the transducer (and in some circumstances, the breakdown of the materials in contact with the transducer) and by the phenomenon of electrostatic pull-in or membrane collapse. Maximum field strengths for oxide and nitride are 800 and $460 \mathrm{~V} / \mu \mathrm{m}$, respectively [18] [20]. If the transducer cavity is not evacuated totally, then gas breakdown may also limit the device configuration. The Paschen effect, which describes the effective increase of breakdown fields as the electrode separation decreases, is not considered quantitatively here. The main point is that a limit to the manipulation of $V_{\mathrm{DC}}, t_{a}$, and $t_{m}$ exists because of electrical breakdown.

Another such limit stems from the possibility of membrane collapse. A simplified expression for the collapse voltage, $V_{\text {collapse }}$, is

$$
V_{\text {collapse }}=\left(\frac{8 \kappa\left(t_{a}+\frac{\epsilon_{0}}{\epsilon} t_{m}\right)^{3}}{27 \epsilon_{0} A}\right)^{\frac{1}{2}}
$$

where $\kappa$ is the spring constant of the transducer membrane. A derivation for this expression is given in Appendix 1. Eq. (4) assumes that uniform electrostatic pressure exists over the entire membrane, which is of questionable validity at the point of collapse, when the center of the deflected membrane is more strongly attracted than the edges. Thus, (4) represents the best case scenario and should be used with caution, especially in the case of partially metalized membranes. For a more robust prediction of $V_{\text {collapse, }}$, we instead use computer simulations as is shown in Section IV.

Assuming that $V_{\mathrm{DC}}, t_{a}$, and $t_{m}$ have been optimized to a practical or theoretical limit, (3) seems to imply that no further optimization is possible. However, the key observation of this paper is that judicious patterning of the electrode metalization results in a transformer ratio $n_{\text {partial }}$ such that $\tau_{\text {partial }}$ is smaller than $\tau_{\text {full }}$. In this case, $(2)$ is replaced by

$$
n_{\text {partial }}=f\left(M(x, y), V_{D C}, t_{a}, t_{m}\right)
$$

where $M(x, y)$ describes the metalization pattern. Eq. (3) can then be rewritten for a circular membrane and metal- 


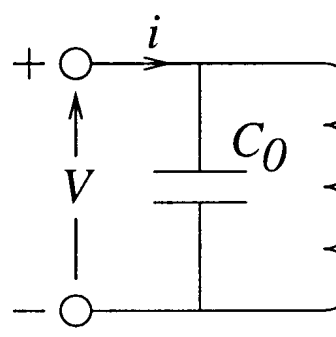

\section{Electrical Port Acoustic Port}

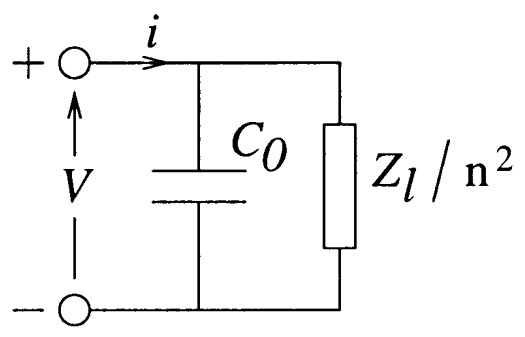

Equivalent Circuit

Fig. 3. Reduced electrical circuit for the cMUT membrane.

ization pattern as

$$
\begin{array}{r}
\tau_{\text {partial }}=\frac{\epsilon_{0} \epsilon S_{\text {electrode }}}{f\left(M(r), V_{D C}, t_{a}, t_{m}\right)^{2}\left(\epsilon_{0} t_{m}+\epsilon t_{a}\right)} \\
Z_{\text {medium }} S_{\text {membrane }}
\end{array}
$$

where $r$ is electrode radius. We do not propose an analytical expansion of (6), but rather use finite element simulations to show that $\tau_{\text {partial }}$ can be made smaller than $\tau_{\text {full }}$.

Qualitatively, the argument is that the transformer ratio relates the current and voltage at the electrical port to the velocity and force at the acoustic port and that its value depends on the electrode pattern. The subtlety lies in the fact that the velocity and force of the acoustic ports are lumped parameters of a system that in reality is distributed. The force, as defined in the derivation of the equivalent circuit, is a uniform force over the entire membrane, and the velocity is defined as the average of the velocities on the contour of the membrane. Given that the electrostatic force is not uniform, especially in the case of partially metalized electrodes, consistency with the equivalent circuit model requires that the lumped electrostatic force ( $F$ in Fig. 2) be interpreted as an effective force. This effective force $\left(F_{\text {effective }}\right)$ is the force that, if applied uniformly over the entire membrane, would give the same peak membrane displacement that the patterned electrode gives. Fig. 4 compares the static deflections of equally biased $25-\mu \mathrm{m}$ radius membranes: one that is fully metalized, one that is centrally metalized at half the radius, and one that is centrally metalized at a quarter of the radius. From Fig. 4, we see that a half metalized membrane and a fully metalized membrane have very similar deflections; so, the lumped effective force associated with the two is the same. Consequently, the half metalized membrane will have broader bandwidth than the fully metalized membrane because its $C_{0}$ is smaller, although its $n$ is essentially the same. To find the electro-mechanical transformer ratio $n$ in a dynamic analysis, one needs to compute the average membrane velocity corresponding to an applied voltage $V_{\mathrm{AC}}$ with no acoustic load, multiply that with $Z_{a}$ to find $F_{\text {effective }}$ and then divide $F_{\text {effective }}$ by the applied voltage:

$$
n_{\text {partial }}=\frac{F_{\text {effective }}}{V_{\mathrm{AC}}}=\frac{v Z_{a}}{V_{\mathrm{AC}}} .
$$

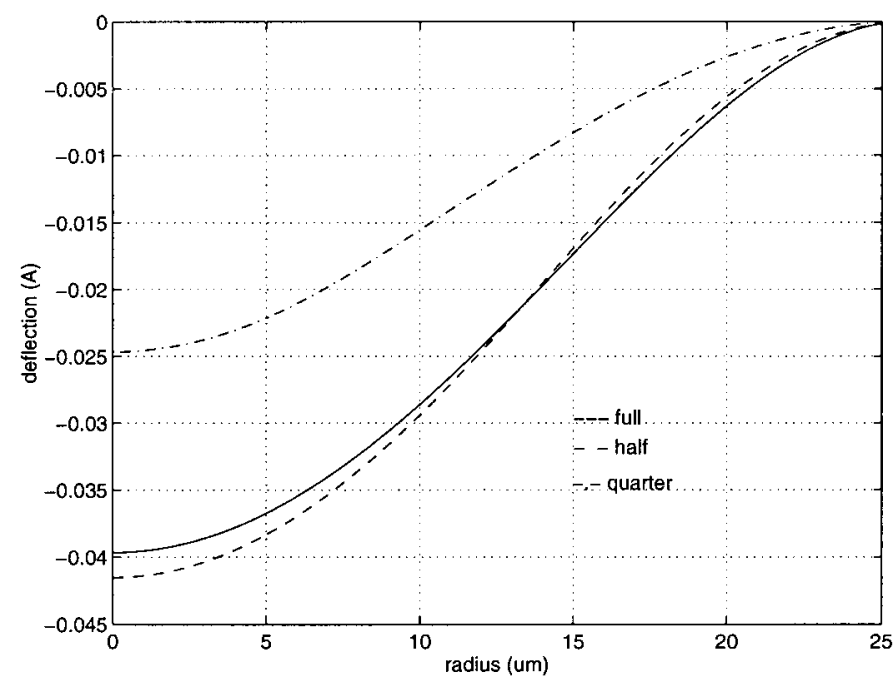

Fig. 4. Deflection of cMUT membrane for various metalization levels.

An important point to note here is that $Z_{a}$ is a mechanical property associated with the cMUT membrane; it is not altered with changing electrode size or with loading. It is also important to note that for the analyses herein presented, the actual value of $Z_{a}$ is not very significant because it is dwarfed by the magnitude of $Z_{l}$; rather, the value of $n_{\text {partial }}$, for a given $Z_{a}$, because of electrode minimization, is important. The following section presents more detail about the finite element simulations and quantitatively demonstrates that electrode patterning can indeed improve cMUT performance in the specific case of circular membranes.

\section{Simulations}

The performance of the partially metalized cMUT device is tested by running simulations using a finite element package (ANSYS Revision 5.2, ANSYS Inc., Canonsburg, PA). In this paper, all analysis is performed for a circular membrane with a centered circular electrode. Because the device is an object of rotation, an axisymmetric model is used. The dimensions of the device are shown in Fig. 5. All of the analyses described below are done for each individual electrode radius.

The analytical paradigm for the simulation begins with the realization that electrostatic forces generated by a volt- 


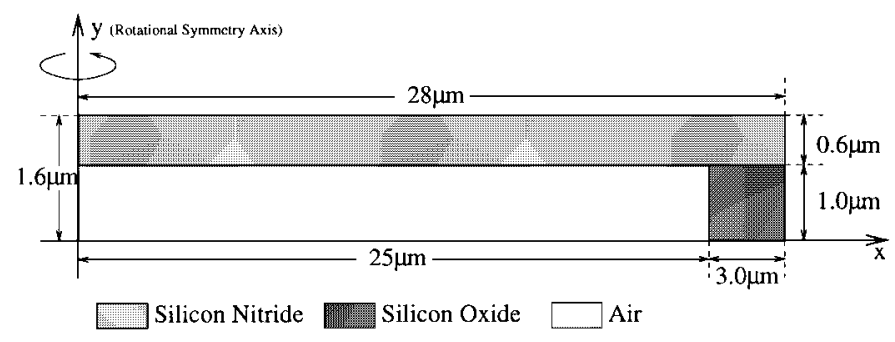

Fig. 5. ANSYS model of circular cMUT.

age are always attractive, regardless of the polarity of the applied voltage. Hence, a voltage of the form

$$
V(t)=V_{\mathrm{DC}}+V_{\mathrm{AC}} \sin \omega t
$$

with $V_{\mathrm{DC}} \gg V_{\mathrm{AC}}$ is applied to the membrane to assure harmonic motion. The applied DC bias $V_{\mathrm{DC}}$ will cause the membrane to deflect toward the substrate. We assume that $V_{\mathrm{AC}}$ results in a small harmonic motion compared with the DC deflection so that the resulting electric field intensity is

$$
E(r, t)=E_{\mathrm{DC}}(r)+E_{\mathrm{AC}}(r) \sin \omega t
$$

where $r$ is the radial distance from the membrane's center, $E_{\mathrm{DC}}(r)$ is the E-field intensity at the membrane-electrode boundary for the DC bias, and

$$
E_{\mathrm{AC}}(r)=\frac{V_{\mathrm{AC}}}{V_{\mathrm{DC}}} E_{\mathrm{DC}}(r)
$$

The electrostatic pressure on the membrane can be found using the Maxwell Stress Tensor equation:

$$
\mathbf{T}(r, t)=\mathbf{n} \frac{\epsilon^{2}}{\epsilon_{0}} \frac{E^{2}(r, t)}{2}
$$

where $\mathbf{n}$ is the surface normal of the metal electrode and $\epsilon$ is the permittivity of the membrane material. Substituting (9) into (11) and ignoring second-order terms yields the temporal and spacial variation of electrostatic pressure on the cMUT surface:

$$
\mathbf{T}(r, t)=\mathbf{n} \frac{\epsilon^{2}}{2 \epsilon_{0}} E_{\mathrm{DC}}^{2}(r)+\mathbf{n} \frac{\epsilon^{2}}{\epsilon_{0}} E_{\mathrm{DC}}(r) E_{\mathrm{AC}}(r) \sin \omega t .
$$

The simulation program is first used to determine the DC deflection of the membrane. The DC solution is then used as an operating point for the harmonic (AC) analysis of the cMUT membrane.

\section{A. Static Analysis-Membrane Deflection}

The static displacement of the membrane is of interest in the determination of the shunt input capacitance, the collapse voltage, and field quantities for the harmonic analysis. All of these require the determination of the membrane shape for an applied DC voltage. First, the Thermal package of ANSYS (see Appendix 2) is used to find the electrostatic field for a certain metalization size. E-field intensity at the electrode-membrane boundary is found by computing the thermal gradient. Then, we employ the Maxwell Stress Tensor equation (11) to find the electrostatic pressure on the membrane:

$$
\mathbf{T}_{\mathrm{DC}}(r)=\mathbf{n} \frac{\epsilon^{2}}{2 \epsilon_{0}} E_{\mathrm{DC}}^{2}(r) .
$$

The electrostatic pressure data of (13) is used as the load in a structural analysis of the device. This analysis yields the membrane shape for the applied load. However, changing the membrane shape changes the electrostatic field solution and, hence, the electrostatic pressure. Thus, new load data have to be determined from the new shape of the membrane. The final shape of the membrane is found iteratively; consecutive electrostatic (thermal) and structural analyses are performed until satisfactory convergence is observed.

1. Static Analysis-Membrane Collapse Voltage: The iteration to determine the membrane shape is terminated either when the membrane shape stabilizes or when the membrane collapses onto the substrate. For the former case, the applied voltage is lower than the collapse voltage; for the latter case, the applied voltage is higher than the collapse voltage. The collapse voltage is the value of the DC bias at which the membrane is infinitesimally close to collapse. Fig. 6 shows iteration results for a stable and collapsing membrane, respectively. The applied voltages are 230 and $250 \mathrm{~V}$. The collapse voltage for this particular membrane is some value between these voltages. The trace labeled "Analytic" is the result of the analytical treatment assuming uniform deflections and forces.

This analysis method has been used to determine the collapse voltage for varying electrode sizes. Fig. 7 shows the simulation results for the device of Fig. 5. Because the total force on the membrane scales with electrode area, a higher voltage is required for the collapse of a membrane with a smaller electrode. The subtle inflection point around $17 \mathrm{~V}$ is explained by the fact that the fringing fields generated by the larger electrode sizes have a smaller component in the direction of collapse.

2. Static Analysis - Input Capacitance: The results of the static analysis are used to determine the shunt input capacitance $C_{0}\left(V_{\mathrm{DC}}\right)^{2}$ of the transducer. For a certain DC bias voltage, the shape of the membrane is determined by the iterative simulation described previously. Once the electric field intensity at the deflected membrane surface is known, the charge distribution on the electrode is found using Gauss' Theorem. The capacitance of the membrane for various electrode sizes is determined by computing the total charge on the membrane and dividing this by the applied voltage. So, if $E_{\mathrm{DC}}(r)$ is the electric field intensity,

\footnotetext{
${ }^{2} C_{0}$ is a function of the $\mathrm{DC}$ bias voltage $V_{\mathrm{DC}}$ because the membrane shape is determined by the bias voltage.
} 

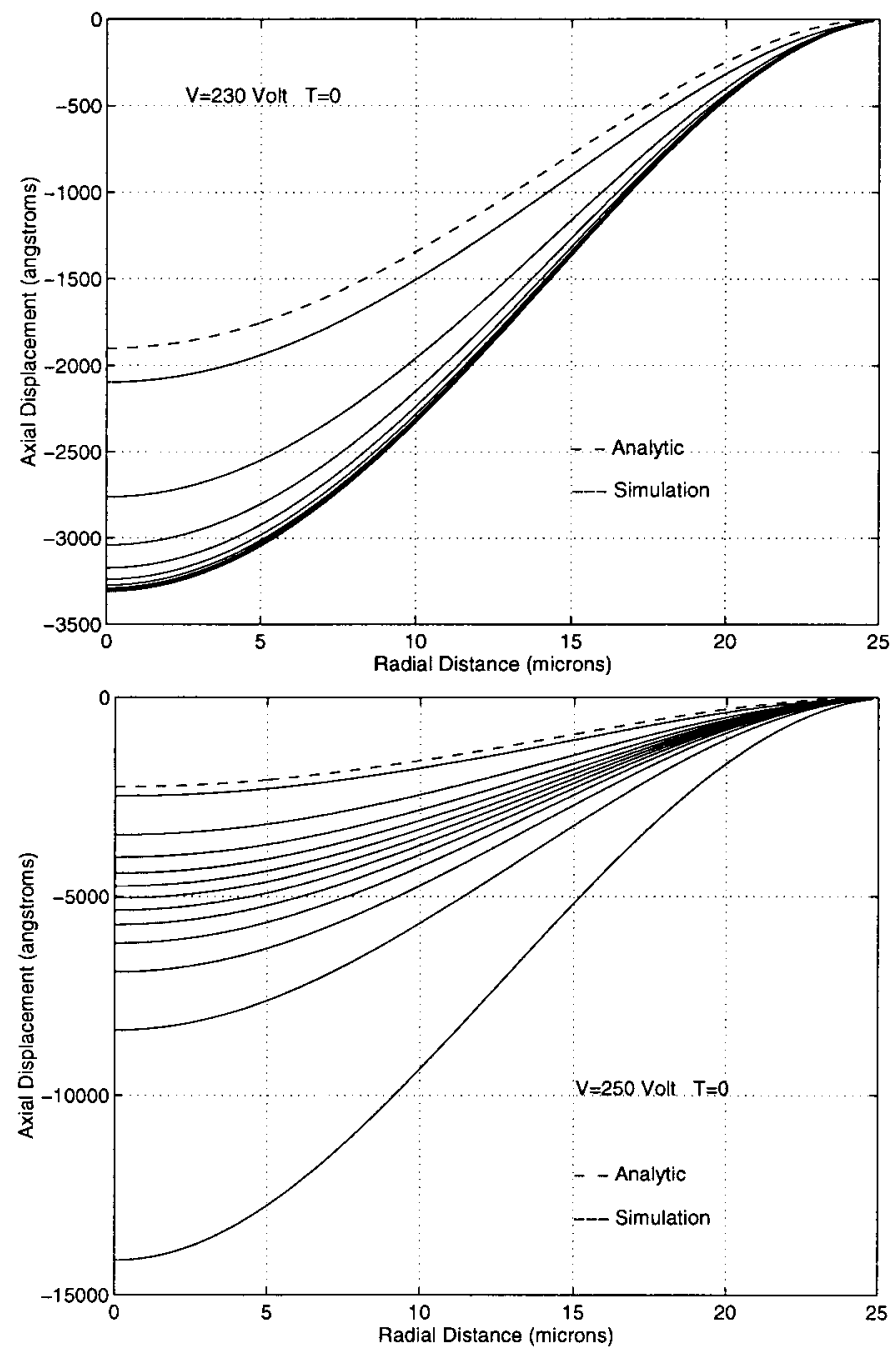

Fig. 6. Iteration results for a stable and a collapsing membrane.

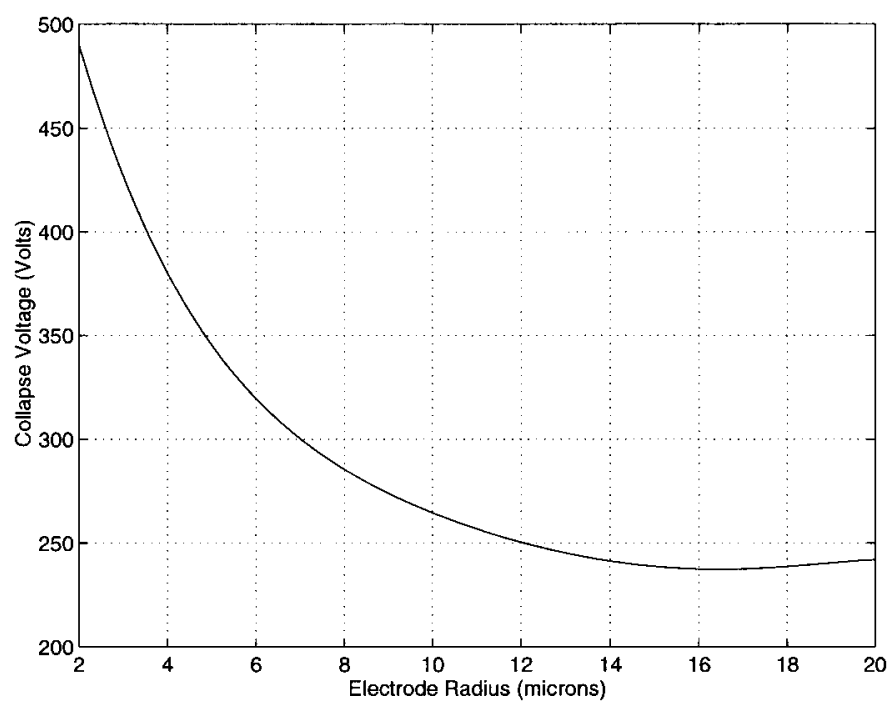

Fig. 7. Collapse voltage values for varying electrode sizes.
$C_{0}\left(V_{\mathrm{DC}}\right)$ is given by

$$
C_{0}\left(V_{\mathrm{DC}}\right)=\frac{\epsilon}{V_{\mathrm{DC}}} \oint_{S} \mathbf{E}_{\mathrm{DC}} \cdot d \mathbf{s}=\frac{2 \pi \epsilon}{V_{\mathrm{DC}}} \int_{0}^{a} r E_{\mathrm{DC}}(r) d r
$$

\section{B. Harmonic Analysis-Bandwidth of cMUT}

The aim of the harmonic analysis is the determination of additional model parameters needed to determine the device bandwidth: the lumped acoustic impedance of the membrane $Z_{a}$ and the electro-mechanical transformer ratio $n . Z_{a}$ is a mechanical property associated with the cMUT membrane and does not change with changing electrode size (if the loading effect of the metal electrode is neglected). Thus, we first find $Z_{a}$ for particular device dimensions and use this value for all subsequent analyses of varying electrode sizes. The electro-mechanical transformer ratio, $n$, is a function of metalization radius and, thus, has to be calculated for each individual electrode size.

1. Harmonic Analysis-Membrane Impedance: The acoustic impedance $Z_{a}$ of the membrane is found by first finding $v(\omega)$ for zero acoustic load (i.e., the cMUT in vacuum) and a uniform excitation pressure at the set of frequencies of interest, then dividing the total force on the membrane by these velocity values. The accuracy of the analysis is tested by comparing the simulated impedance values to analytical results. In both the simulated and the analytical solution, the metalization electrode is neglected, and only the nitride membrane is considered. Such an approximation is justified because the electrode is usually much thinner than the nitride (four times thinner in a practical device). When the relevant physical quantities of the electrode material, such as density and Young's modulus, are considered, it can be shown that the electrode is negligible for the purposes of the analyses in this paper. Mason [17], in his formulation of the membrane's mechanical behavior, assumes that the membrane ends are clamped. The same boundary conditions are imposed during simulations for test purposes. For the stated boundary conditions, there is a remarkable match between the analytical and numerical results. The actual membrane's ends are not clamped; rather, they rest on the sacrificial oxide layer. Simulation results for this case show that the resonance frequency of the actual membrane is slightly less than that of the clamped membrane. Fig. 8 shows the two simulation results together with the analytic impedance curve.

2. Harmonic Analysis-Partially Metalized cMUT: Once the lumped acoustic impedance $Z_{a}$ of the cMUT membrane is found, we can find the electromechanical transformer ratio for each individual electrode size by finding the average membrane velocity under harmonic excitation with zero acoustic load (i.e., vacuum). Here, we assume that the DC deflection of the membrane is large compared with the harmonic component of the movement 


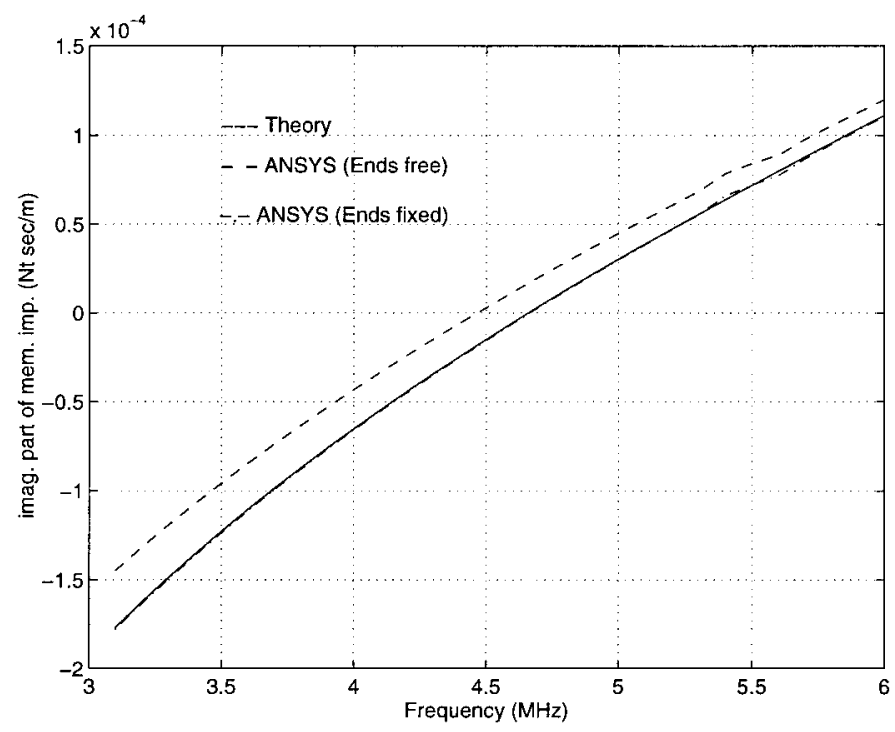

Fig. 8. Acoustic impedance of membrane with zero residual stress.

so that the AC behavior can be analyzed separately from the DC behavior. The key assumption is that mechanical properties of the membrane, such as Poisson's ratio and Young's modulus, are not perturbed by the DC deflection. Hence, the undeflected $Z_{a}$ is still valid, and DC and AC displacements can be computed separately and summed to yield the overall movement of the membrane. In other words, if

$$
T(r, t)=T_{\mathrm{DC}}(r)+T_{\mathrm{AC}}(r) \sin \omega t
$$

with

$$
T_{\mathrm{DC}}(r)=\frac{\epsilon^{2}}{2 \epsilon_{0}} E_{\mathrm{DC}}^{2}(r) ; \quad T_{\mathrm{AC}}(r)=\frac{\epsilon^{2}}{\epsilon_{0}} E_{\mathrm{DC}}(r) E_{\mathrm{AC}}(r)
$$

from (12), then membrane deflection is given by

$$
x(r, t)=x_{\mathrm{DC}}(r) \pm x_{\mathrm{AC}}(r) \sin (\omega t+\phi(\omega))
$$

where $x_{\mathrm{DC}}(r)$ is the DC deflection of the membrane and $x_{\mathrm{AC}}(r)$ is the time harmonic component of the membrane motion around the bias point and $\phi(\omega)$ is the phase angle ${ }^{3}$ of the membrane's motion at a frequency $\omega$. Combining (10) and (16):

$$
T_{\mathrm{AC}}(r)=2 \frac{V_{\mathrm{AC}}}{V_{\mathrm{DC}}} T_{\mathrm{DC}}(r)
$$

where $T_{\mathrm{DC}}(r)$ is the $\mathrm{DC}$ pressure on the cMUT membrane that causes it to deflect to its operating point. Consequently, the harmonic solution $x_{\mathrm{AC}}(r)$ can be found by applying $T_{\mathrm{AC}}(r)$ to the DC-deflected membrane surface.

The displacement values found from the harmonic analysis are used to find the average membrane velocity by

\footnotetext{
${ }^{3}$ For $\omega<\omega_{c}$, the membrane impedance is greatly determined by its stiffness; thus, $x_{\mathrm{AC}}$ is in-phase with $T_{\mathrm{AC}}$. For $\omega>\omega_{c}$, the influence of the membrane mass becomes more significant; therefore, $x_{\mathrm{AC}}$ and $T_{\mathrm{AC}}$ are $180^{\circ}$ out-of-phase.
}

evaluating the subsequent integral:

$$
v(\omega)=\frac{1}{A} \int_{0}^{a} 2 \pi r \omega x_{\mathrm{AC}}(r) d r .
$$

Multiplying $v(\omega)$ with $Z_{a}(\omega)$ yields the effective total force $F_{\text {effective }}(\omega)$ on the cMUT membrane. The electromechanical transformer ratio is then given by

$$
n=\frac{F_{\text {effective }}(\omega)}{V_{\mathrm{AC}}}
$$

It should be pointed out that $n$ has a negligible frequency dependence as long as only the primary vibration mode of the membrane is possible. For verification, simulations were run at two frequencies (one smaller than $\omega_{c}$ and the other greater), and the same values for $n$ were found.

\section{Results}

The electro-mechanical transformer ratios for various electrode sizes are determined by running structural simulations and computing the average membrane velocity under harmonic excitation. These results are used to find the effective force on the membrane and, consequently, the electro-mechanical transformer ratios. In Fig. 9, plots of $n, 1 / n^{2}, C_{0}$, and the bandwidth of the resulting RC network, which is $1 / \tau_{\text {partial }}$, are given. The figure graphically describes equations (5) and (6). The last graph indicates that with the proposed criteria, a transducer of dimensions shown in Fig. 5, is optimized by an electrode with an $11 \mu \mathrm{m}$ radius.

For the purpose of electrical matching, we select a lossless matching network topology to tune out the parasitic element $\left(C_{0}\right)$ of the transducer equivalent circuit [21], [22]. The sixth-order maximally flat (Butterworth) network [23] shown in Fig. 10 is used for the electrical matching. $C_{0}$ is set equal to the shunt input capacitance of the transducer, the source resistance is chosen as equal to the radiation resistance of the transducer, and the center frequency of the network is set to the mechanical resonance frequency of the membrane. The remaining component values are computed by properly scaling the values in the prototype network of [23], which are also found in various Butterworth tables of radio handbooks. The resulting bandwidth of the transducer for two different metal electrode sizes is depicted in Fig. 11. For both electrode sizes, the DC bias voltage is assumed to be $200 \mathrm{~V}$. This is less than the voltage that causes the fully metalized membrane to collapse, which was found to be $240 \mathrm{~V}$ by simulations. This value for the collapse voltage is almost one-half the value found using the analytic expression of (4).

As found by simulations, the collapse voltage of the membrane increases by decreasing electrode size. If the DC bias is set to the collapse voltage, the bandwidth of the transducer further increases for smaller electrodes. Fig. 12 shows the bandwidth of the transducer of Fig. 5 as a function of electrode radius when the bias is set to the collapse voltages shown in Fig. 7. According to this result, 

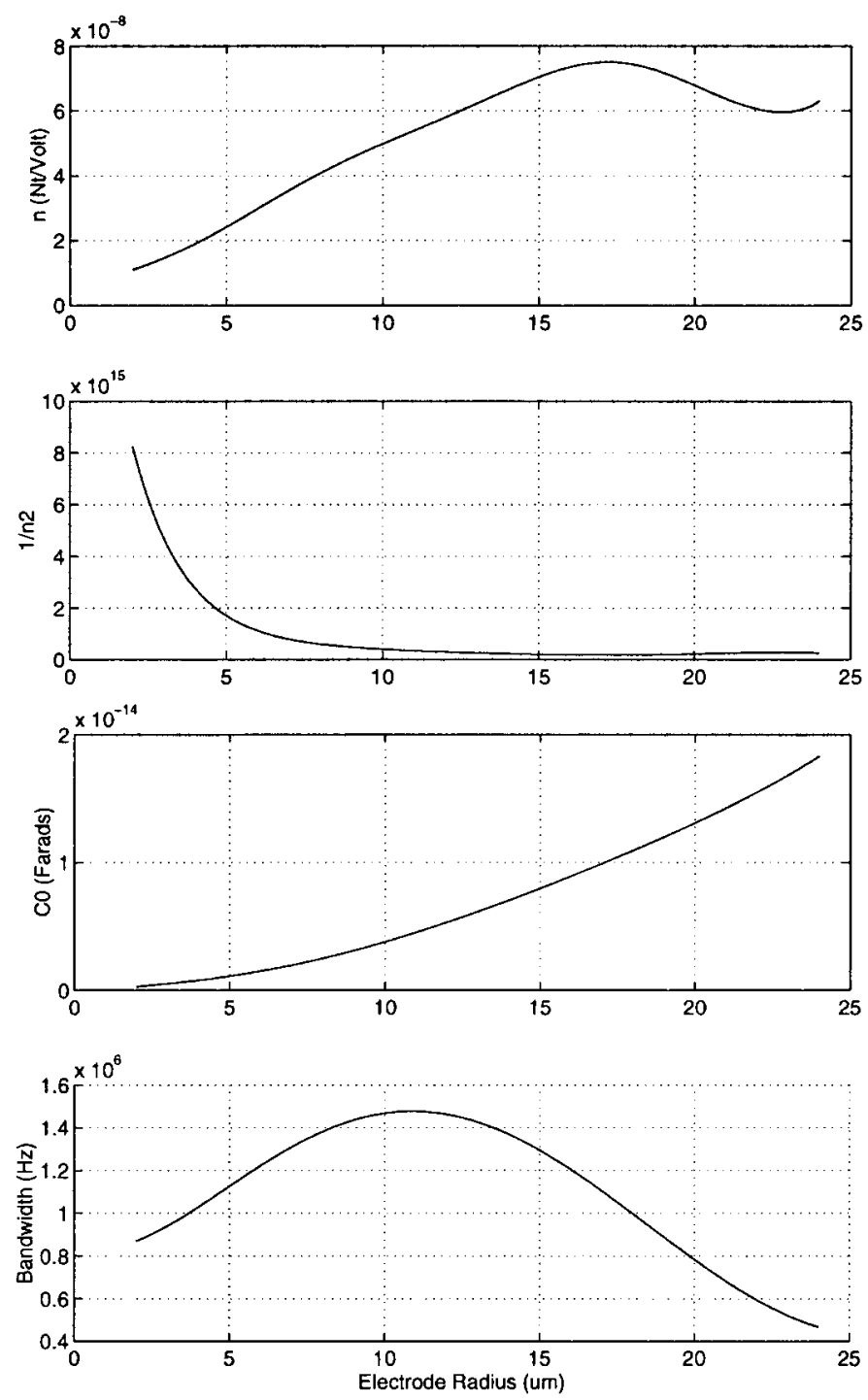

Fig. 9. Electro-mechanical transformer ratio, capacitance and bandwidth of cMUT transducer for electrode radius ranging from 2 to $24 \mu \mathrm{m}$.

the transducer with maximum bandwidth should have a top electrode as small as possible. Interconnections to the top electrode will set a limit on how small it can get, as will breakdown mechanisms.

Numerous parameters are involved in the design of a transducer with maximum bandwidth. As shown in Appendix 3 , for bias voltages close to the collapse voltage, the effect of changing the air gap $t_{a}$ is negligible. However, the bandwidth of the cMUT linearly increases with membrane thickness $t_{n}$. The practical usefulness of this observation is limited, though, because the collapse voltage is proportional to $t_{n}^{3}$, which implies that thicker membranes, in order to operate near $V_{\text {collapse }}$, require very high bias voltages. Furthermore, as $t_{n}$ increases beyond a critical value, a major assumption of the analyses, that $Z_{l} \gg Z_{a}$, is violated, and the acoustic impedance of the membrane begins to dominate the transduction dynamics. Hence, given a desired operating frequency and a practical bias voltage

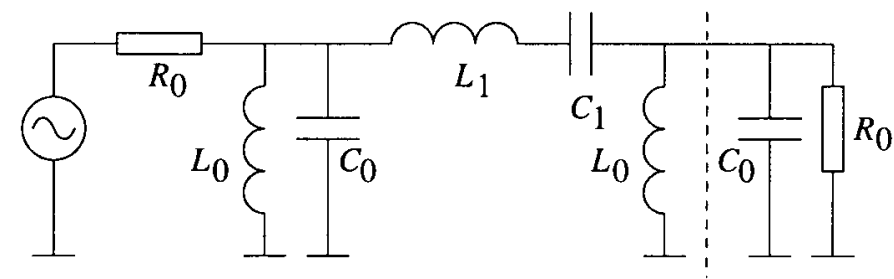

Fig. 10. Butterworth network for electrical matching.

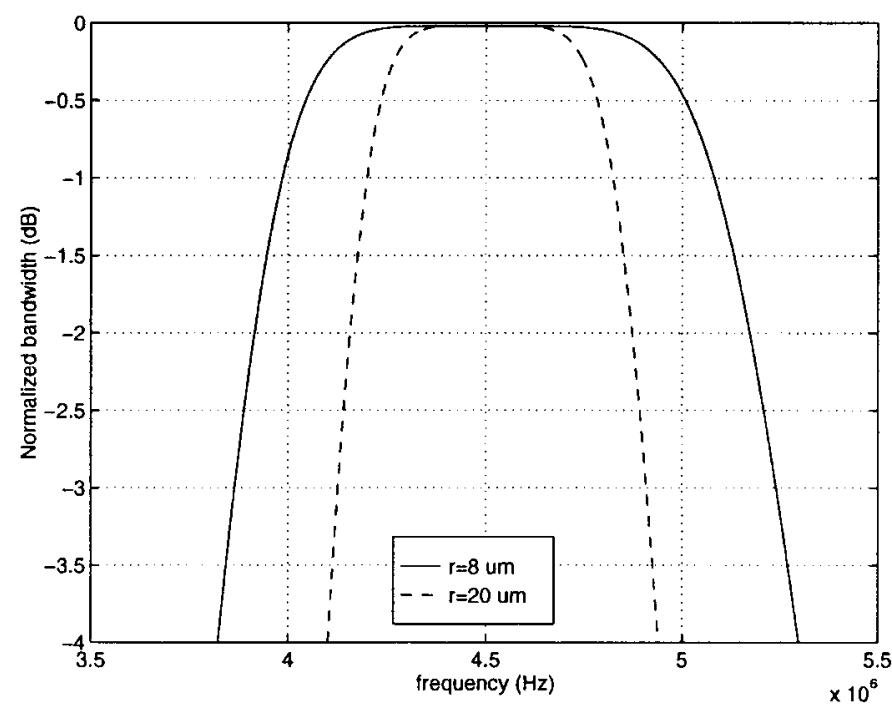

Fig. 11. Normalized transducer bandwidth for two electrode sizes.

limit, the membrane should be made as thick as possible, so long as the transduction dynamics are still dominated by the load impedance. This paper demonstrates that further improvement to bandwidth is achieved by adjusting the electrode radius. The simulation results shown in Fig. 11 show that the bandwidth can be increased by a factor of about 2 when a smaller top electrode is used. The optimum metalization radius ranges between 40 and $50 \%$ of the membrane radius and needs to be determined individually for each transducer of different dimensions. These results are for a constant bias voltage value. ${ }^{4}$ Similar analyses were done for a transducer with dimensions $r=50 \mu \mathrm{m}, t_{n}=2.5 \mu \mathrm{m}$ and $t_{a}=1.0 \mu \mathrm{m}$ to find that an electrode of radius $23 \mu \mathrm{m}$ yields maximum bandwidth for a fixed bias voltage.

The harmonic simulation of the membrane not only shows that electrode optimization can increase bandwidth but also demonstrates that the membrane deflection caused by the DC bias voltage works to increase device bandwidth. Electrostatic forces are significantly higher for a deflected membrane than for an unbiased membrane. Hence, the electromechanical conversion efficiency is found higher than when bias induced deflection is ignored. However, the shunt input capacitance is higher as well because the electrode separation is reduced. The subtlety lies in re-

\footnotetext{
${ }^{4}$ As Fig. 7 and 12 show, with a smaller electrode, the membrane can withstand a larger DC bias value, which further increased the bandwidth.
} 
TABLE I

Collapse Voltage, Source Resistance, and Bandwidth as a Function of Electrode Radius for a Transducer Having Dimensions $t_{n}=0.3 \mu \mathrm{M}, t_{a}=0.5 \mu \mathrm{M}$, AND $a=17.7 \mu \mathrm{M}$. Source Resistance is Computed for a 2 -CM $\times 2$-CM Device. The Mechanical Resonance is at $4.5 \mathrm{MHz}$.

\begin{tabular}{cccc}
\hline $\begin{array}{c}\text { Electrode } \\
\text { Radius } \\
a(\mu \mathrm{m})\end{array}$ & $\begin{array}{c}\text { Collapse } \\
\text { Voltage } \\
V_{\text {collapse }}(\mathrm{V})\end{array}$ & $\begin{array}{c}\text { Source } \\
\text { Resistance } \\
R_{0}(\mathrm{k} \Omega)\end{array}$ & $\begin{array}{c}\text { Bandwidth } \\
1 / \tau(\mathrm{MHz})\end{array}$ \\
\hline 1.4142 & 160 & 0.746 & 3.682 \\
2.8284 & 110 & 0.406 & 2.350 \\
4.2426 & 88 & 0.245 & 1.957 \\
5.6569 & 75 & 0.206 & 1.477 \\
7.0711 & 68 & 0.153 & 1.331 \\
8.4853 & 63 & 0.116 & 1.251 \\
11.3137 & 60 & 0.093 & 0.953 \\
14.1422 & 60 & 0.071 & 0.833 \\
16.9706 & 60 & 0.092 & 0.494 \\
\hline
\end{tabular}

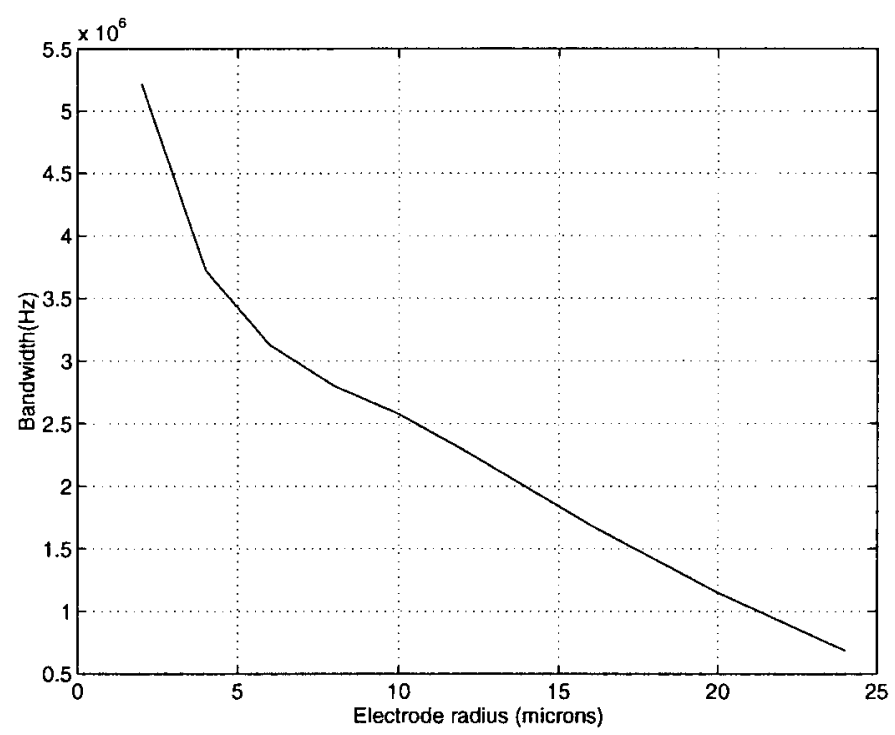

Fig. 12. Transducer bandwidth for DC bias equal to the collapse voltage.

alizing that the decrease in the acoustic load impedance as transformed to the electrical port has a square dependence on the separation distance (electrostatic force changes with the inverse square of the distance), and capacitance has a linear dependence. Therefore, the bandwidth of the cMUT found from simulations accounting for the bias-induced deflection is about two times larger than the bandwidth when the DC deflection is ignored.

\section{A. Design Example}

Table I shows simulation results for a sample transducer. For a metal electrode radius of $4.24 \mu \mathrm{m}$, the transducer has a collapse voltage of $88 \mathrm{~V}$. The DC bias voltage is set to the collapse voltage. The resulting transducer bandwidth is $1.96 \mathrm{MHz}$ (43\%) for lossless matching. The

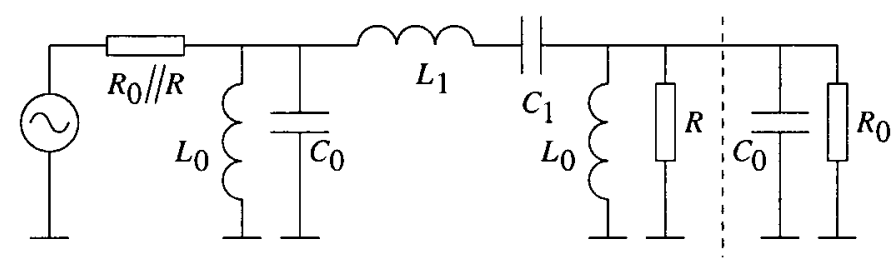

Fig. 13. Lossy matching network.

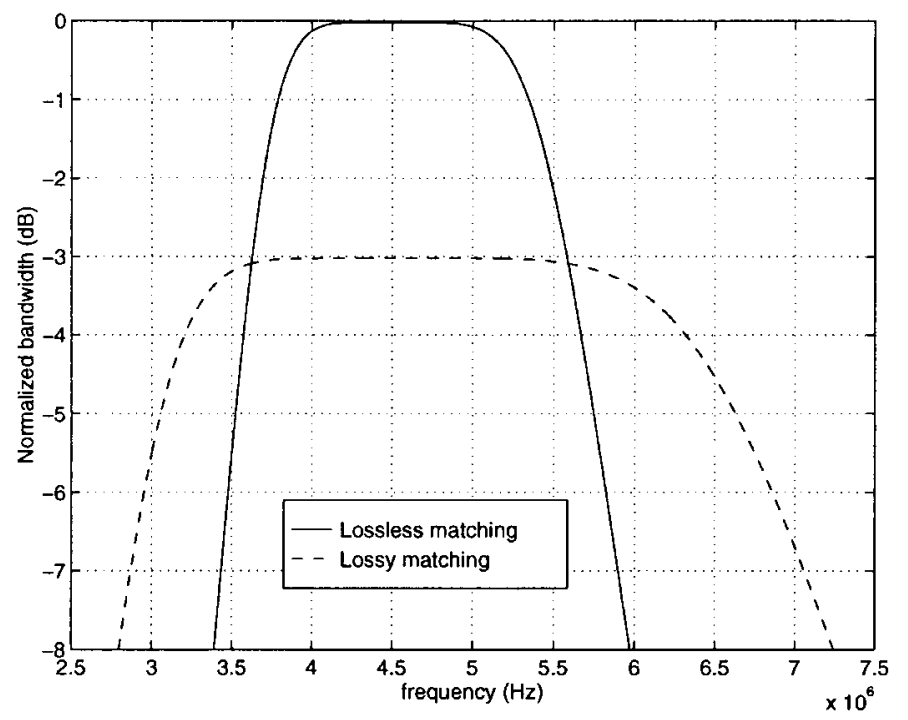

Fig. 14. Transducer bandwidth for lossless and lossy matching networks.

bandwidth can be doubled at the expense of $50 \%$ power loss by using the matching network depicted in Fig. 13 with an additional shunt resistor $R$ equal to $R_{0}$, which is employed to reduce $\tau$. As expected with this network, the bandwidth is increased to $3.91 \mathrm{MHz}(87 \%)$. The transducer bandwidth for both matching networks is shown in Fig. 14.

The total source resistance $R_{0}$ of a multi-element device is determined by the number of elements that constitute the transducer. Table I lists source resistance values for a device size of $2 \mathrm{~cm} \times 2 \mathrm{~cm}$. The value of $R_{0}$ is relevant to system design, where impedance matching allows for the best noise performance. Here, any additional transducer loss is ignored and for each electrode radius, the bias voltage is set to the corresponding collapse voltage. All DC bias voltages are smaller than the conservatively estimated breakdown voltage of $524 \mathrm{~V}$.

\section{CONCLUSION}

We have demonstrated that the optimization objective for cMUTs is to minimize the time constant of the RC circuit formed by the capacitance of the device $C_{0}$ and the transformed acoustic load $Z_{l}$. Given practical and theoretical limitations on thickness dimensions and DC voltages, a method has been proposed to improve device bandwidth further by judiciously patterning the membrane electrode. 
Finite element computer simulations have been used to demonstrate that, in the case of circular immersion cMUT membranes, an electrode 40 to $50 \%$ the size of the membrane increases bandwidth by a factor of 2.5 . In a specific design example, we have shown that a $43 \% 3$-dB bandwidth is feasible; $87 \%$ is realized with an additional $6 \mathrm{~dB}$ of insertion loss. The principles behind these specific results imply that future cMUT designs will incorporate electrode patterning to enhance performance in both immersion and gas-coupled applications. Current research efforts include the generation of devices with a patterned electrode and consideration of the second-order effects that the electrode pattern may have on diaphragm vibrations.

\section{Appendix 1}

When the DC bias voltage applied to the membrane exceeds a critical value, the membrane collapses over the silicon membrane. This critical voltage ( $\left.V_{\text {collapse }}\right)$ can be found by modeling the membrane as a parallel plate capacitor suspended above a fixed ground plate with a linear spring. The spring constant $(\kappa)$ can be found as the ratio of pressure to volume displacement: [17]

$$
\kappa=\frac{T A t_{n}}{\left(\frac{c}{d}-\frac{a}{2} \frac{J_{0}(a \sqrt{d / c})}{J_{1}(a \sqrt{d / c})} \sqrt{\frac{c}{d}}+\frac{a^{2}}{8}\right)}
$$

where

$$
c=\frac{(E+T) t_{n}^{2}}{12 \rho\left(1-\sigma^{2}\right)} ; d=\frac{T}{\rho}
$$

where $T, \rho$, and $\sigma$ are the residual stress, density, and Poisson's ratio of the membrane material, respectively, and $A$ is the area of the membrane. If $x$ denotes the membrane displacement, the total restoring string force is

$$
F_{S}=\kappa x
$$

The electrostatic force on the membrane is given by

$$
F_{E}=\frac{A \epsilon^{2} V^{2}}{2 \epsilon_{0}\left(t_{n}+\frac{\epsilon}{\epsilon_{0}}\left(t_{a}-x\right)\right)^{2}} .
$$

The voltage to keep the membrane at a certain deflection $x$ can be found by equating $F_{E}$ to $F_{S}$ and solving for $V$. The critical voltage at which the membrane becomes unstable can be determined by finding the displacement for which $\partial V / \partial x=0$. Solving yields

$$
x=\frac{1}{3}\left(t_{a}+\frac{\epsilon_{0}}{\epsilon} t_{n}\right)
$$

and the corresponding collapse voltage is found as

$$
V_{\text {collapse }}=\sqrt{\frac{8 \kappa\left(t_{a}+\frac{\epsilon_{0}}{\epsilon} t_{n}\right)^{3}}{27 A \epsilon_{0}}} .
$$

TABLE II

Analogous Field and Material Quantities.

\begin{tabular}{ll}
\hline Thermal & Electrical \\
\hline$k$ (conductivity) & $\epsilon$ (permittivity) \\
$T$ (temperature) & $\phi$ (scalar potential) \\
$\dot{q}$ (heat generation) & $\rho$ (charge density) \\
$\nabla T$ (temperature gradient) & $-E$ (electric field intensity) \\
\hline
\end{tabular}

\section{APPENDIX 2}

ANSYS, the software used in the Finite Element Method (FEM) simulations, is able to solve static thermal problems, but does not directly support electro-magnetic field solutions. However, thermal packages can be directly employed in the solution of electrostatic problems because of the analogy between their governing differential equations. The thermal Poisson's equation is

$$
\nabla^{2} T=-\dot{q} / k, \text { and }
$$

the electrostatic Poisson's equation is given as

$$
\nabla^{2} \phi=-\rho / \epsilon
$$

which suggests analogous field quantities shown in Table II [24].

\section{APPENDIX 3}

If the cMUT transducer is assumed to be a parallel plate capacitor, its capacitance is

$$
C_{0}=\frac{\epsilon A}{\left(t_{n}+\frac{\epsilon}{\epsilon_{0}} t_{a}\right)} .
$$

For small deflections of the membrane,

$$
E(r, t)=V(t) /\left(t_{n}+\frac{\epsilon}{\epsilon_{0}} t_{a}\right)
$$

Thus,

$$
\begin{aligned}
T(r, t)=\frac{1}{2} & \frac{\epsilon^{2}}{\epsilon_{0}}\left(V_{\mathrm{DC}}^{2}(r)\right. \\
& \left.+2 V_{\mathrm{DC}}(r) V_{\mathrm{AC}}(r) \sin \omega t\right) /\left(t_{n}+\frac{\epsilon}{\epsilon_{0}} t_{a}\right)^{2},
\end{aligned}
$$

and the electro-mechanical transformer ratio $n$, which is the time-varying part of $T(r, t)$ times the membrane area divided by the AC voltage, is

$$
n=A \frac{\epsilon^{2}}{\epsilon_{0}} V_{\mathrm{DC}} \frac{1}{\left(t_{n}+\frac{\epsilon}{\epsilon_{0}} t_{a}\right)^{2}} .
$$

Consequently, the $\mathrm{RC}$ time constant $\tau$ of the transducer, which is given as $\tau=C_{0} Z_{l} / n^{2}$, is

$$
\tau=\frac{\epsilon_{0}^{2}}{\epsilon^{3}} \frac{1}{V_{\mathrm{DC}}^{2}}\left(t_{n}+\frac{\epsilon}{\epsilon_{0}} t_{a}\right)^{3} Z_{l} .
$$


Eq. (26) contains the spring constant $\kappa$ of the membrane as a term, which has an approximate expression [17]

$$
\kappa=\frac{16 \pi Y_{0} t_{n}^{3}}{\left(1-\rho^{2}\right) a^{2}} .
$$

Substituting this into the collapse voltage expression of (26), we get

$$
V_{\text {collapse }}=\sqrt{\frac{128 Y_{0} t_{n}^{3}\left(t_{n}+\frac{\epsilon}{\epsilon_{0}} t_{a}\right)^{3}}{27 \epsilon_{0}\left(1-\rho^{2}\right) a^{4}}} .
$$

Combining (33) and (35) yields the expression for the time constant

$$
\tau=Z_{w} \frac{27\left(1-\rho^{2}\right)}{128 Y_{0}} \frac{a^{4}}{t_{n}^{3}}
$$

where $Z_{w}$ is the acoustic impedance of the loading medium. This equation shows that the bandwidth of the cMUT does not depend on the air gap thickness when $V_{\mathrm{DC}}$ is at $V_{\text {collapse }}$.

The resonance frequency $f_{c}$ of the cMUT membrane is $[17]$

$$
f_{c}=\frac{(2.4)^{2}}{2 \pi} \sqrt{\frac{Y_{0}}{12 \rho\left(1-\delta^{2}\right)}} \frac{t_{n}}{a^{2}} .
$$

If a cMUT is to operate at a certain frequency, $t_{n} / a^{2}$ has to be constant when adjusting device dimensions to increase bandwidth. This condition, when combined with (36) implies that the device bandwidth linearly increases with increasing membrane thickness $t_{n}$.

\section{REFERENCES}

[1] W. Kuhl, G. R. Schodder, and F. K. Schodder, "Condenser transmitters and microphones with solid dielectric for airborne ultrasonics," Acustica, vol. 4, no. 1, pp. 520-532, 1954.

[2] K. Matzuwa, "Capacitive ultrasonic transducer," J. Phys. Soc. Jpn., vol. 13, pp. 1533-1543, 1958.

[3] J. H. Cantrell and J. S. Heyman, "Broadband electrostatic acoustic transducer for ultrasonic measurements in liquids," Rev. Sci. Instrum., vol. 50, pp. 31-33, Jan. 1979.

[4] I. Ladabaum, X. C. Jin, H. T. Soh, A. Atalar, and B. T. KhuriYakub, "Surface micromachined capacitive ultrasonic transducers," IEEE Trans. Ultrason., Ferroelect., Freq. Contr., vol. 45, no. 3, pp. 678-690, 1998.

[5] H. T. Soh, I. Ladabaum, A. Atalar, C. F. Quate, and B. T. Khuri-Yakub, "Silicon micromachined ultrasonic immersion transducers," Appl. Phys. Lett., vol. 69, pp. 3674-3676, Dec. 1996.

[6] I. Ladabaum, B. T. Khuri-Yakub, and D. Spoliansky, "Micromachined ultrasonic transducers: $11.4 \mathrm{MHz}$ transmission in air and more," Appl. Phys. Lett., vol. 68, pp. 7-9, Jan. 1996.

[7] M. I. Haller, "Micromachined ultrasonic devices and materials," Ph.D. dissertation, Stanford Univ., Stanford, CA, 1997.

[8] D. W. Schindel, D. A. Hutchins, L. Zou, and M. Sayer, "The design and characterization of micromachined air-coupled capacitance transducers," IEEE Trans. Ultrason., Ferroelect., Freq. Contr., vol. 42. pp. 42-50, Jan. 1995.

[9] P. Eccardt, K. Niederer, T. Scheiter, and C. Hierold, "Surface micromachined ultrasound transducers in CMOS technology," in Proc. Ultrason. Symp., San Antonio, TX, 1996, pp. 959-962.
[10] K. Suzuki, K. Higuchi, and H. Tanigawa, "A silicon electrostatic ultrasonic transducer," IEEE Trans. Ultrason., Ferroelect., Freq. Contr., vol. 36, pp. 620-627, Nov. 1989.

[11] M. Rafiq and C. Wykes, "The performance of capacitive ultrasonic transducers using V-grooved backplates," Meas. Sci. Technol., vol. 2, pp. 168-174, Feb. 1991.

[12] M. Pentti, F. Tsuzuki, H. Vaataja, and K. Sasaki, "Electroacoustic model for electrostatic ultrasonic transducers with V-grooved backplates," IEEE Trans. Ultrason., Ferroelect., Freq. Contr., vol. 42, pp. 1-7, Jan. 1995.

[13] M. J. Anderson, J. A. Hill, C. M. Fortunko, N. S. Dogan, and R. D. Moore, "Broadband electrostatic transducers: Modeling and experiements," J. Acoust. Soc. Amer., vol. 97, pp. 262-272, Jan. 1995.

[14] A. Prak, M. Elwenspoek, and J. H. Fluitman, "Selective mode excitation and detection of micromachined resonators," in Proc. Micro Electro Mechanical Systems Workshop, Travemunde, Germany, 1992, pp. 220-225.

[15] J. A. Voorthuyzen, A. J. Sprenkels, A.G.H. Van Der Onk, P. R. Scheeper, and P. Bergveld, "Optimization of capacitive microphone and pressure sensor performance by capacitor-electrode shaping," Sensors Actuators A (Physical), vol. 25-27, pp. 331336, 1991.

[16] X. Jin, I. Ladabaum, and B. T. Khuri-Yakub, "The microfabrication of capacitive ultrasonic transducers," in Proc. Transducers 97, Chicago, IL, pp. 436-440.

[17] W. P. Mason, Electromechanical Transducers and Wave Filters. New York, NY: Van Nostrand, 1942.

[18] B. Tareev, Physics of Dielectric Materials. Moscow: Mir Publishers, 1979.

[19] T. S. Moss, Handbook on Semiconductors. New York, NY: North-Holland, 1992.

[20] A. S. Grove, Physics and Technology of Semiconductor Devices. John Wiley \& Sons, Inc., 1967.

[21] D. F. Tuttle, Jr., Electric Networks. McGraw-Hill Book Company, 1965.

[22] R. M. Fano, "Theoretical limitations on the broadband matching of arbitrary impedances," J. Franklin Inst., pp. 57-83, 139-154, Jan. and Feb. 1950.

[23] G. L. Matthaei, Microwave Filters, Impedance Matching Networks, and Coupling Structures. Dedham, MA: Artech House, 1980.

[24] D. F. Ostergaard, "Adapting available finite element heat transfer problems to solve 2-d and 3-d electrostatic field problems," J. Electrostat., vol. 19, pp. 151-164, 1987.

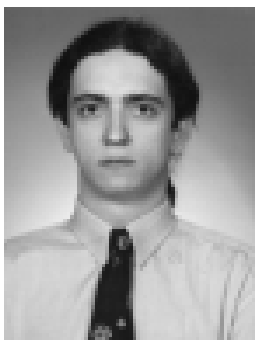

Ayhan Bozkurt was born in Aschasfenburg, Germany, in 1970. He received his B.S. and M.S. from Bilkent University in 1992 and 1994, respectively, both in Electrical and Electronics Engineering. He is about to finish his Ph.D. study at Bilkent University in the Electrical and Electronics Engineering Department.

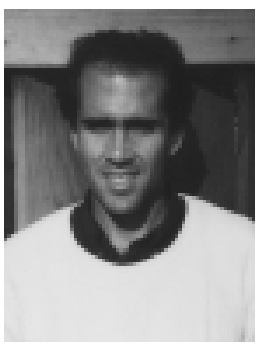

Igal Ladabaum received a B.S. in Bioengineering from U.C. Berkeley in 1992. He then went to Paris, France, where he was a Jean Monnet scholar (1992-1993) at the Ecole Polytechnique. He was also a staff engineer at Air Liquide. In 1996, he received an M.S. in Electrical Engineering from Stanford University. Mr. Ladabaum is interested in the techniques of micromachining and their application to the realization of novel transducers. Most of his effort is directed toward the development and application of ultrasonic transducers. He is a member of the IEEE, the AAAS, the New York Academy of Sciences, and the Acoustical Society of America. He has received numerous awards through the course of his studies, including three best student paper prizes. He has contributed several journal and 
conference papers, and is pursuing patents for some of his work on ultrasonic transducers. Mr. Ladabaum is a co-founder and currently president of Sensant Corp. At the time of submission for press, he was still awaiting official conferral of his Ph.D. degree in Electrical Engineering from Stanford University.

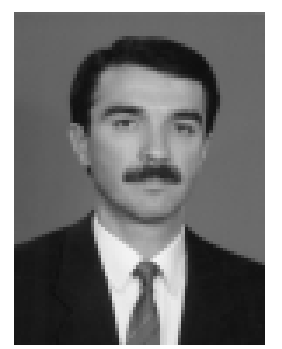

Abdullah Atalar (M'88-SM'90) was born in Gaziantep, Turkey, in 1954. He received B.S. degree from Middle East Technical University, in 1974, M.S. and Ph.D. degrees from Stanford University in 1976 and 1978, respectively, all in Electrical Engineering. His thesis work was on reflection acoustic microscopy. From 1978 to 1980 , he was first a Post Doctoral Fellow and later an Engineering Research Associate at Stanford University, continuing his work on acoustic microscopy. For 8 mo, he was with Hewlett Packard Labs, Palo Alto, engaged in photoacoustics research. From 1980 to 1986, he was on the faculty of the Middle East Technical University as an Assistant Professor. From 1982 to 1983, on leave from the University, he was with Ernst Leitz Wetzlar, West Germany, where he was involved in the development of the commercial acoustic microscope. In 1986, he joined the Bilkent University as chairman of the Electrical and Electronics Engineering Department and served in the founding of the Department, where he is now a professor. He is presently the Provost of Bilkent University. He teaches undergraduate and graduate courses on VLSI design and microwave electronics. His current research interests include micromachined sensors and actuators and computer-aided design in Electrical Engineering. He is the project director of a NATO SFS project: TU-MIMIC. He is a senior member of IEEE.

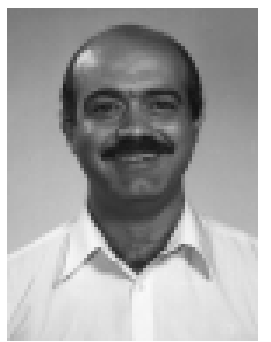

Butrus T. Khuri-Yakub (S'70-S'73-M'76SM'87-F'95) was born in Beirut, Lebanon. He received the B.S. degree in 1970 from the American University of Beirut, the M.S. degree in 1972 from Dartmouth College, and the Ph.D. degree in 1975 from Stanford University, all in electrical engineering. He joined the research staff at the E. L. Ginzton Laboratory of Stanford University in 1976 as a research associate. He was promoted to a Senior Research Associate in 1978 and to a Professor of Electrical Engineering (Research) in 1982. He has served on many university committees such as graduate admissions, undergraduate academic council of the school of engineering, and others. He has been teaching both at the graduate and undergraduate levels for over $15 \mathrm{yr}$, and his current research interests include in situ acoustic sensors (temperature, film thickness, resist cure, etc.) for monitoring and control of integrated circuits manufacturing processes, micromachining silicon to make acoustic materials and devices such as air-borne and water immersion ultrasonic transducers and arrays and fluid ejectors, and the field of ultrasonic NDE and acoustic imaging and microscopy.

Professor Khuri-Yakub is a fellow of the IEEE, a senior member of the Acoustical Society of America, and a member of Tau Beta Pi. $\mathrm{He}$ is Associate Editor of Research in Nondestructive Evaluation, a Journal of the American Society for Nondestructive Testing, and a member of the AdCom of the IEEE group on Ultrasonics Ferroelectrics and Frequency Control (January 1994 to January 1997). He has authored about 300 publications and has been principal inventor or coinventor on over 30 patents. He received the Stanford University School of Engineering Distinguished Advisor Award (June 1987) and the Medal of the City of Bordeaux for contributions to NDE (1983). 